\title{
Estimating Radiation Exposure in Interventional Environments
}

\author{
Alexander Ladikos ${ }^{1}$, Cedric Cagniart ${ }^{1}$, Reza Ghotbi ${ }^{2}$, \\ Maximilian Reiser ${ }^{3}$, and Nassir Navab ${ }^{1}$ \\ ${ }^{1}$ Chair for Computer Aided Medical Procedures, Technische Universität München \\ ${ }^{2}$ Center for Vascular and Endovascular Surgery, Klinikum Pasing, München \\ ${ }^{3}$ Department of Clinical Radiology, Ludwig-Maximilians-Universität München
}

\begin{abstract}
In the last decade the use of interventional X-ray imaging, especially for fluoroscopy-guided procedures, has increased dramatically. Due to this the radiation exposure of the medical staff has also increased. Although radiation protection measures such as lead vests are used there are still unprotected regions, most notably the hands and the head. Over time these regions can receive significant amounts of radiation. In this paper we propose a system for approximating the radiation exposure of a physician during surgery. The goal is to sensibilize physicians to their radiation exposure and to give them a tool to quickly check it. To this end we use a real-time $3 \mathrm{D}$ reconstruction system which builds a $3 \mathrm{D}$-representation of all the objects in the room. The reconstructed 3Drepresentation of the physician is then tracked over time and at each time step in which the X-Ray source is used the radiation received by each body part is accumulated. To simulate the radiation we use a physicsbased simulation package. The physician can review his radiation exposure after the intervention and use the collected radiation information over a longer time period in order to minimize his radiation exposure by adjusting his positioning relative to the X-ray source. The system can also be used as an awareness tool for less experienced physicians.
\end{abstract}

\section{Introduction}

In contrast to even 10 years ago the use of interventional X-ray imaging and therefore the dosage received by the physician has increased dramatically, especially for fluoroscopy-guided procedures. In fluoroscopy the X-ray source may be turned on for more than 30-60 minutes [10 during which the physician is standing very close to the patient and therefore the X-ray source. Due to the increase in the use of fluoroscopy-guided procedures, interventional radiologists have nowadays a much greater risk of radiation damage. Although the dose received in a single intervention is not large, it is well known, that long-term radiation exposure even at low doses can lead to negative effects on the body, which in the extreme can lead to cell mutations and cancer. The physician is usually protected by a lead vest and sometimes a lead collar around his neck. However, this leaves some body parts still exposed to the radiation, most notably

T. Jiang et al. (Eds.): MICCAI 2010, Part III, LNCS 6363, pp. 237 244, 2010.
(C) Springer-Verlag Berlin Heidelberg 2010 
the head, the arms and the hands. Especially the hands are at danger since they are used to work on the patient and therefore closest to the radiation source 1015 . To control the radiation received a dosimeter is typically worn under the lead-vest. However, since the dosimeter is worn under the protective vest, its readings are not representative of the radiation received by the unprotected body parts. Moreover since it is placed at the level of the chest it is further away from the X-ray source than for instance the hands and therefore only of limited use. We therefore want to present a solution to physicians, especially the less experienced ones, which allows them to quickly gauge their radiation exposure during an intervention and over the course of a longer time frame, in order to sensibilize them for the dangers associated with X-ray radiation and the options of limiting their exposure. To this end we propose a real-time 3D-reconstruction system combined with a geometric tracking algorithm which allows us to reconstruct and track the physician in the interventional room during the procedure and - with the knowledge about the position and movement of the X-ray source - to approximate his radiation exposure.

Contributions. The contributions of this paper are twofold: On the technical side we present a powerful methodology for tracking the physician from his realtime 3D-reconstruction and for modeling the scatter radiation created by the $\mathrm{X}$-ray source. The tracking is a non-trivial problem since the physician has to be tracked based only on his geometry and correspondences have to be established between corresponding body parts in different reconstructions. The simulation of the scatter radiation is done using a physics framework for simulating particle physics [5]. In the simulation we model the C-arm and the part of the patient irradiated considering physical interaction effects such as Compton Scattering, Rayleigh Scattering and the Photoelectric Effect. The simulation results are used to determine the radiation deposited in an object at a given position relative to the C-arm, such as the physician.

On the medical side we show a concept for a system which sensibilizes the physician to his radiation exposure and which helps him to take more informed decisions when using X-ray devices to minimize his risk for radiation related dangers. The system displays a color-coded map of the physician after each intervention which shows his radiation exposure during the procedure and if desired the accumulated radiation for a given time frame. This allows the physician to keep track of his long-term radiation exposure. The system can also be used for making inexperienced physicians more aware of the dangers of radiation exposure.

\section{Related Work}

There have been many medical studies concerning the radiation exposure of patients and surgeons during different interventions [131156]. In [15] for instance the radiation exposure of the patient and the surgeon is modeled mathematically. However, only estimates about the average distance of the surgeon to X-ray source are used. Many studies conclude that the radiation exposure of the 
physician during fluoroscopy-guided interventions is very high in the area of the hands [10] and in the lower extremities [13. This has sparked some discussion about the possibility of protecting the surgeon and making him more aware of the dose he has already received. Our system attacks at this point since it allows the physician to check his exposure easily in an everyday setting.

There has also been work on simulating the radiation exposure for training novel physicians [16]2]. In this work the C-arm was modeled in a simulation and the radiation dose was measured at several spherical detectors around the $\mathrm{C}$ arm. However, the position of the physician was not taken into account and the system was not meant for use in interventional rooms but for training. We are not aware of any work which tracks the physician during the intervention and which accumulates his radiation exposure over the course of an intervention.

On the technical side, which builds the foundation of our system, there has been a lot of work which was mainly published in the computer vision literature. For the real-time 3D-reconstruction of the scene we use the visual hull algorithm described in [7]. The visual hull [9] is an approximation of the true shape of an object which is computed from silhouette images by intersecting the silhouettebackprojections. The recovered shape is the shape maximally consistent with the input silhouette images.

The tracking stage of our system is responsible for establishing dense correspondences between the independent 3D-reconstructions of the scene computed at each time frame. As we are interested in the movement of the physician, our work relates directly to the vast body of literature addressing markerless human motion-capture in multi-camera environments. Most of these vision-based tracking algorithms use kinematic models in the form of a skeleton as discussed in the survey by Moeslund et al. [11]. Such skeletal models allow to effectively constrain the problem and reduce its dimensionality but they usually require manual initialization of the joint positions, as well as lengthy parametrization of the tracked person's body characteristics from generic mesh models. In contrast to these approaches, we chose to use the framework recently presented by [3]. This algorithm doesn't rely on strong priors on the nature of the tracked object and instead deforms the first reconstructed mesh to fit the geometry in the rest of the sequence.

\section{Reconstruction and Tracking System}

We use a multi-camera system [8] to reconstruct the shape of the objects inside the interventional room in real-time. The system consists of 16 optical cameras mounted on the ceiling as well as a few PCs to perform the reconstruction and the radiation modeling (see Figure 11). In an offline phase, the initial position of the C-arm inside the reconstruction volume is determined and the cameras are calibrated. In addition, background images of the room are acquired. In the online phase, the background images are used to segment the foreground objects. The segmented images are subsequently used to perform the 3D-reconstruction by intersecting the backprojections of the object silhouettes in space. The reconstruction of the physician is then tracked using a mesh deformation framework. 

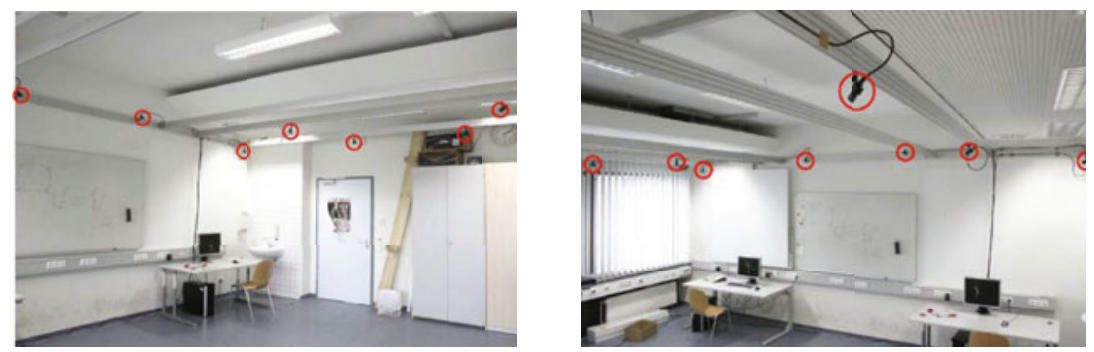

Fig. 1. View of our reconstruction studio. The cameras are marked by red circles.

In a final step, the scatter radiation created by the $\mathrm{C}$-arm is modeled and accumulated using the reconstruction of the physician. Once the system has been set up, very little maintenance work is required. In the following sections we will describe each component of the system in more detail.

\subsection{System Architecture}

We use a distributed system for performing the 3D-reconstruction. A total of four PCs with four cameras each is connected through a Gigabit-Ethernet network. The PCs acquire the camera images, perform the background subtraction using the method presented in [4] and then perform a partial 3D-reconstruction using their locally available silhouette images 7 . The results are sent to a master $\mathrm{PC}$ which combines the partial reconstructions and performs the radiation accumulation. The results are then visualized.

We decided to place the cameras on the ceiling so that they do not take away any workspace from the operating room and to protect them from accidental collisions with other objects, which would require a recalibration of the system. The cameras are synchronized in order to perform a consistent reconstruction.

\subsection{Calibration}

Before the system can be used the cameras have to be calibrated extrinsically and intrinsically. To this end we use a calibration toolbox designed for multicamera environments [14]. It is based on a calibration point which is moved around in the room and detected in the cameras. The method is easy to use and delivers an accurate calibration. Typical calibration times are in the range of 15 minutes. The recovered camera coordinate system is registered to the room coordinate system using target points at known positions in the room.

\subsection{Segmentation}

It is necessary to extract the foreground objects in the camera images in order to perform the 3D-reconstruction. This is done using a robust background subtraction method 4]. Reference images are acquired before the intervention 
and are used during runtime in order to detect the changed parts in the scene. This results in the silhouette images which are the input for the reconstruction. In the case of static foreground objects, such as a surgical table, problems can arise, because these objects will occlude other objects which in turn cannot be fully reconstructed since they are not fully seen in the silhouette images. We solve this problem by masking out static objects in the silhouette images, effectively declaring them to foreground. This automatically adds them to the reconstruction.

\subsection{Reconstruction}

The silhouette images are used in a GPU-based visual hull computation method 7. First the reconstruction volume is discretized into voxels. Each voxel is then checked for its occupancy by projecting it into all available silhouette images. If it is empty in at least one of the silhouette images it is marked as empty, otherwise it is marked as occupied. In order to speed this up an octree model of the reconstruction volume is used and the computations are streamlined on the GPU.

\subsection{Tracking}

The result of the 3D-reconstruction stage is a sequence of meshes independently reconstructed at each time frame. We use the non-rigid mesh registration framework presented by [3] to iteratively deform a reference mesh to fit the geometry in the rest of the sequence. The reference mesh is taken from the first frame of the sequence. We prefer this tracking method to skeletal-based alternatives as it does not need an initialization of the pose and of the body characteristics of the tracked person. The only requirement is that the tracked physician must be clearly distinct from the rest of the reconstructed geometry in the reference frame. The output of the tracking algorithm is a dense trajectory for each vertex of the reference mesh across the sequence, which is necessary to accumulate the radiation exposure over time.

\section{Radiation Modeling}

We model the scatter radiation created by the interaction of the X-rays with the patient's body using the particle-physics simulation system GEANT4 [5]. Using this system it is possible to model the different physical effects, which lead to the production of scatter radiation, namely Compton Scattering, Rayleigh Scattering and the Photoelectric Effect. We model the scene similar to [16. In that work the authors also use the GEANT4 system to model X-ray scatter radiation. We model the $\mathrm{C}$-arm as an intensifier tube and a detector both made out of iron. This has the effect that radiation is blocked above the detector and below the source. The patient is modeled as a cylinder containing water. As has been shown in $16 \mid 2$ the model, although simplified from reality, still provides 
reasonable simulation results which make it useful for educating physicians about their radiation exposure. After having modeled the $\mathrm{C}$-arm and the patient we create a X-ray beam originating from the radiation source and direct it in a narrow cone at the patient body. In order to obtain the energy deposited by the $\mathrm{X}$-rays in the environment we place a so-called detector sphere around the scene in our simulation and register the energy of the particles which fall onto it. By placing several such spheres with different radii and all centered on the source of the scatter radiation, i.e. the patient, we can estimate the distribution of the radiation inside the room and thereby the radiation received by the physician. We precompute an X-ray irradiation volume using the settings of the C-arm and compose it with the tracked mesh in the previous step. This allows us to accumulate the radiation received by each vertex and by interpolation the radiation received by the whole mesh.

\section{Results}

To validate our system we recorded an interventional scenario in our lab consisting of a C-arm, a patient and a physician. The physician is moving around
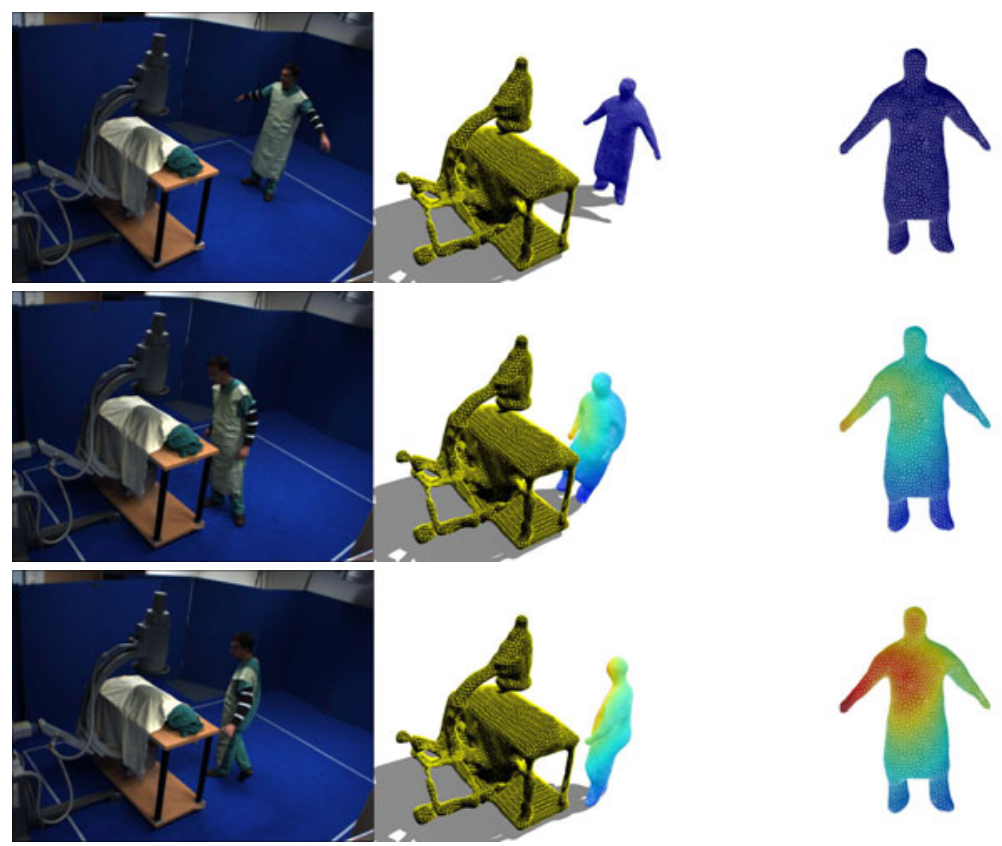

Fig. 2. Results on a sequence recorded in our lab. The three rows show the radiation exposure at the beginning, the middle and the end of the intervention. The first column shows one of the input images, the second column shows the tracked 3D-scene and the final column shows the physician in his reference pose with the color-coded radiation exposure. Red represents a high radiation exposure while blue represents a low radiation exposure. 
the $\mathrm{C}$-arm while the $\mathrm{C}$-arm is constantly radiating. This is a typical scenario for a fluoroscopy-guided intervention, since in fluoroscopy the C-arm is also almost constantly radiating. The frame rate at which the reconstruction system is running is $20 \mathrm{fps}$. Once we obtain the reconstruction of the physician, we start the tracking algorithm. The physician is then tracked during the entire sequence. With the knowledge of the position of the X-ray source and the patient we can compute the source of the scatter radiation which is subsequently computed using the radiation simulation framework. Since we have the tracked mesh of the surgeon with corresponding vertices over time, we can simply add up the radiation at each vertex position for each frame. By performing this addition over the whole sequence we obtain the final radiation dose collected by the physician for each vertex of his reconstruction. These values are then interpolated to obtain the radiation on the whole mesh. This is reasonable since the mesh consists of only small triangles. Finally, we visualize the accumulated radiation using a heat map (see Figure 2 and the videos included as additional material). The scaling on the heat map is set so that the maximum amount of radiation received by the physician is marked as bright red. This makes it easier to visually gauge the exposure. It can be seen, as also observed in [15], that the hands receive most of the radiation.

\section{Discussion}

We understand our system as a proof-of-concept. Our goal was not to develop a system which can be directly used in an operating room, but to show what problems have to be addressed, which methods are available to solve them and how they can be combined in a sensible way. In particular the exposure computed by our system needs to be validated by experimental measurements and the dynamic and static environment of the interventional room needs to be taken into account in more detail. The use of our system is not limited to the estimation of the radiation exposure. The availability of a real-time 3D-reconstruction of the interventional environment can also be used for workflow analysis [12, collision detection [8] and documentation purposes.

\section{Conclusion}

We presented a system for modeling the radiation received by a physician during an intervention. Our system builds on a real-time 3D-reconstruction of the interventional room which is used for tracking the 3D-mesh of the physician using a mesh deformation framework. The radiation is modeled using a particle-physics simulation package. The contribution of the paper is the combination of realtime 3D-reconstruction and mesh-based tracking to help the physician estimate his radiation exposure and to allow him to collect statistics about his long-term exposure. Our system can also be used for making novice physicians more aware of their radiation exposure. Future work includes validating the radiation estimation by using sensors attached to the physician, creating profiles for each physician and bringing the system to a real operating room. 


\section{References}

1. Bogaert, E., Bacher, K., Thierens, H.: A large-scale multicentre study in belgium of dose area product values and effective doses in interventional cardiology using contemporary x-ray equipment. Radiation Protection Dosimetry 128(3), 312-323 (2008)

2. Bott, O., Wagner, M., Duwenkamp, C., Hellrung, N., Dressing, K.: Improving education on c-arm operation and radiation protection with a computer-based training and simulation system. International Journal of Computer Assisted Radiology and Surgery 4(4), 399-407 (2009)

3. Cagniart, C., Boyer, E., Ilic, S.: Probabilistic deformable surface tracking from multiple videos. In: ECCV (2010)

4. Fukui, S., Iwahori, Y., Itoh, H.i., Kawanaka, H., Woodham, R.: Robust background subtraction for quick illumination changes. In: Chang, L.-W., Lie, W.-N. (eds.) PSIVT 2006. LNCS, vol. 4319, pp. 1244-1253. Springer, Heidelberg (2006)

5. GEANT4, http://www. cern.ch/geant4

6. Giordano, B.D., Baumhauer, J.F., Morgan, T.L., Rechtine, G.R.: Patient and surgeon radiation exposure: Comparison of standard and mini-c-arm fluoroscopy. Journal of Bone \& Joint Surgery 91(2), 297-304 (2009)

7. Ladikos, A., Benhimane, S., Navab, N.: Efficient visual hull computation for realtime 3d-reconstruction using cuda. In: Proceedings of the 2008 Conference on Computer Vision and Pattern Recognition Workshops (2008)

8. Ladikos, A., Benhimane, S., Navab, N.: Real-time 3d reconstruction for collision avoidance in interventional environments. In: Metaxas, D., Axel, L., Fichtinger, G., Székely, G. (eds.) MICCAI 2008, Part II. LNCS, vol. 5242, pp. 526-534. Springer, Heidelberg (2008)

9. Laurentini, A.: The visual hull concept for silhouette-based image understanding. PAMI 16(2), 150-162 (1994)

10. Marx, M., Ellis, J.: Radiation protection of the hand in interventional radiology: should it fit like a glove? Radiology 200(1), 24-25 (1996)

11. Moeslund, T.B., Hilton, A., Krueger, V.: A survey of advances in vision-based human motion capture and analysis. Comput. Vis. Image Underst. 104(2), 90-126 (2006)

12. Padoy, N., Blum, T., Essa, I., Feussner, H., Berger, M.-O., Navab, N.: A boosted segmentation method for surgical workflow analysis. In: Ayache, N., Ourselin, S., Maeder, A. (eds.) MICCAI 2007, Part I. LNCS, vol. 4791, pp. 102-109. Springer, Heidelberg (2007)

13. Shortt, C.P., Al-Hashimi, H., Malone, L., Lee, M.J.: Staff radiation doses to the lower extremities in interventional radiology. Cardiovascular Interventional Radiology 30, 1206-1209 (2007)

14. Svoboda, T., Martinec, D., Pajdla, T.: A convenient multi-camera self-calibration for virtual environments. Presence: Teleoperators and Virtual Environments 14(4), 407-422 (2005)

15. Tsalafoutas, I., Tsapaki, V., Kaliakmanis, A., Pneumaticos, S., Tsoronis, F., Koulentianos, E., Papachristou, G.: Estimation of radiation doses to patients and surgeons from various fluoroscopically guided orthopedic surgeries. Radiation Protection Dosimetry 128(1), 112-119 (2008)

16. Wagner, M., Duwenkamp, C., Dresing, K., Bott, O.J.: An approach to calculate and visualize intraoperative scattered radiation exposure. In: MIE (2009) 